\title{
3 Research Square

\section{The structure of SpoT reveals evolutionary tuning of enzymatic output through constraint of the conformational landscape}

\section{Hedvig Tamman}

Université Libre de Bruxelles https://orcid.org/0000-0003-4453-7814

\section{Karin Ernits}

Lund University

\section{Mohammad Roghanian}

Lund University

\section{Andres Ainelo}

Université Libre de Bruxelles

\section{Christina Julius}

Umea University

\section{Anthony Perrier}

University of Namur https://orcid.org/0000-0002-4473-3711

\section{Ariel Talavera}

Vrije Universiteit Brussel, Vlaams Instituut voor Biotechnologie https://orcid.org/0000-0002-1865-5959

\section{Hanna Ainelo}

Université Libre de Bruxelles

\section{Rémy Dugauquier}

Université https://orcid.org/0000-0002-9662-8905

\section{Safia Zedek}

Université Libre de Bruxelles

\section{Aurélien Thureau}

Swing Beamline, Synchrotron SOLEIL https://orcid.org/0000-0001-5666-260X

Javier Perez

Synchrotron SOLEIL https://orcid.org/0000-0003-3083-4754

\section{Gipsi Lima-Mendez}

University of Namur

\section{Regis Hallez}

University of Namur https://orcid.org/0000-0003-1175-8565

\section{Gemma Atkinson}

Umeå University https://orcid.org/0000-0002-4861-4584

Vasili Hauryliuk 
Lund University https://orcid.org/0000-0003-2389-5057

Abel Garcia-Pino ( $\nabla$ agarciap@ulb.ac.be )

Université Libre de Bruxelles https://orcid.org/0000-0002-0634-0300

\section{Article}

Keywords: (p)ppGpp, stringent response, SpoT, RelA, Rel, allostery, intrinsically disordered 46 proteins, energetic frustration, metabolic hubs, conformational switches

Posted Date: February 11th, 2022

DOI: https://doi.org/10.21203/rs.3.rs-1293174/v1

License: (c) (1) This work is licensed under a Creative Commons Attribution 4.0 International License. Read Full License 
1 The structure of SpoT reveals evolutionary tuning of enzymatic output

2 through constraint of the conformational landscape

Hedvig Tamman ${ }^{1, \dagger, *}$, Karin Ernits ${ }^{2,3,4, \dagger}$, Mohammad Roghanian ${ }^{2,4,5, \dagger}$, Andres Ainelo ${ }^{1}$, Christina Julius $^{4}$, Anthony Perrier ${ }^{6,7}$, Ariel Talavera ${ }^{1}$, Hanna Ainelo ${ }^{1}$, Rémy Dugauquier ${ }^{1,5}$, Safia Zedek ${ }^{1}$, Aurelien Thureau ${ }^{8}$, Javier Pérez ${ }^{8}$, Gipsi Lima-Mendez ${ }^{6}$, Regis Hallez ${ }^{6,7,9}$, Gemma C. Atkinson $^{2,4}$, Vasili Hauryliuk ${ }^{2,4,10, *}$, Abel Garcia-Pino ${ }^{1,9, *}$

${ }^{1}$ Cellular and Molecular Microbiology, Faculté des Sciences, Université libre de Bruxelles (ULB), Boulevard du Triomphe, Building BC, (1C4 203), 1050 Brussels, Belgium

${ }^{2}$ Department of Experimental Medicine, University of Lund, 22184 Lund, Sweden

${ }^{3}$ Department of Chemistry, Umeå University, 90187 Umeå, Sweden

${ }^{4}$ Department of Molecular Biology, Umeå University, 90187 Umeå, Sweden

${ }^{5}$ Departement of Clinical Microbiology, Rigshospitalet, 2200 Copenhagen, Denmark

${ }^{6}$ Biology of Microorganisms Research Unit (URBM), Namur Research Institute for Life Science (NARILIS), University of Namur, 61 Rue de Bruxelles, 5000 Namur

${ }^{7}$ Bacterial Cell cycle \& Development (BCcD), Biology of Microorganisms Research Unit (URBM), Namur Research Institute for Life Science (NARILIS), University of Namur, 61 Rue de Bruxelles, 5000 Namur

${ }^{8}$ Synchrotron SOLEIL, Saint-Aubin - BP 48, 91192 Gif sur Yvette Cedex, France

${ }^{9}$ WELBIO, Avenue Hippocrate 75, 1200 Brussels, Belgium

${ }^{10}$ University of Tartu, Institute of Technology, 50411 Tartu, Estonia

* to whom correspondence should be addressed:

Hedvig Tamman: hedvig.tamman@ulb.be, +372 7376038

Vasili Hauryliuk: vasili.hauryliuk@med.lu.se, +46 706090493

Abel Garcia-Pino: abel.garcia.pino@ulb.be, +32 26505377

†These authors contributed equally to the paper as first authors.

\section{Abstract:}

Stringent factors orchestrate bacterial cell reprogramming through increasing the level of the alarmones (p)ppGpp. In Beta- and Gammaproteobacteria, SpoT hydrolyses (p)ppGpp to counteract the synthetase activity of RelA. However, structural information about how SpoT controls the levels of (p)ppGpp is missing. Here we present the crystal structure of the hydrolase-only SpoT from Acinetobacter baumannii and uncover the mechanism of intramolecular regulation of "long"-RSH factors. In contrast to ribosome-associated Rel/RelA that adopt an elongated structure, SpoT assumes a compact $\tau$-shaped structure in which the regulatory domains wrap around a Core subdomain that controls the conformational state of the enzyme. The Core is key to the specialisation of long-RSHs towards either synthesis or hydrolysis: while the short and structured Core of SpoT stabilises the $\tau$-state priming the HD domain for (p)ppGpp hydrolysis, the longer, more dynamic Core of RelA destabilises the $\tau$ state precluding (p)ppGpp hydrolysis and priming RelA for synthesis.

Keywords: (p)ppGpp, stringent response, SpoT, RelA, Rel, allostery, intrinsically disordered proteins, energetic frustration, metabolic hubs, conformational switches 


\section{Introduction}

RelA-SpoT Homolog (RSH) stringent factors regulate virtually all aspects of bacterial physiology by controlling the levels of the signalling nucleotide alarmones guanosine pentaphosphate and tetraphosphate, collectively referred to as (p)ppGpp ${ }^{1-6}$. The ribosomeassociated "long" multidomain RSH RelA is a dedicated amino acid starvation sensor with a strong (p)ppGpp synthesis activity (SYNTH) that is induced upon detection of deacylated tRNA in the ribosomal A site ${ }^{7,8}$, and no detectable hydrolase activity ${ }^{9}$. In the cell the SYNTH activity of RelA is balanced by SpoT RSH, a bifunctional enzyme with a strong, strictly $\mathrm{Mn}^{2+}$ dependent hydrolase (HD) activity ${ }^{10,11}$ and weak SYNTH activity ${ }^{12}$. The RelA-SpoT pair is a product of gene duplication of an ancestral factor - the ribosome-associated bifunctional RSH Rel - and the pair is limited in its taxonomic distribution to Beta- and Gammaproteobacteria 1,13 .

Subfunctionalisation - the partitioning of functions between two paralogues that arose through gene duplication - appears to have happened at least twice in Gammaproteobacteria (Fig. 1a). First, relatively soon after the duplication that gave rise to RelA and SpoT, RelA lost its capacity for alarmone hydrolysis, evolving into a monofunctional, SYNTH-only RSH. Secondly, as evidenced by a lack of sequence conservation in sites that are critical for nucleotide pyrophosphorylation, during the evolution of the Moraxellaceae lineage of Protobacteria, SpoT has likely lost its synthetase function (Fig. 1a-b) ${ }^{1}$. This resulted in further specialization into mono-functional (p)ppGpp hydrolase, SpoT[Hs] (The uppercase " $\mathrm{H}$ " stands for hydrolasecompetent, while the lowercase "s" indicates "synthetase-incompetent"), as opposed to the bifunctional HD- and SYNTH-competent SpoT[HS] found in other Beta- and Gammaproteobacteria. Recent studies of the Moraxellaceae representative A. baumannii - the " $A$ " in the ESKAPE group of human pathogens of particular concern - indicate a lack (p)ppGpp in the $\Delta r e l A$ strain, both with and without acute amino acid starvation induced by serine hydroxamate (SHX) ${ }^{14,15}$. These observations are consistent with the hypothesis that RelA is, indeed, the sole source of the alarmone in this bacterium. Furthermore, consistent with the key role of (p)ppGpp-mediated signalling in bacterial virulence and antibiotic tolerance ${ }^{16,17}$, the likely $\mathrm{ppGpp}^{0}$ A. baumannii $\Delta$ relA strain displays increased sensitivity to multiple antibiotics 14,15 , decreased virulence in a Galleria mellonella wax moth model and deficiency in switching from the virulent opaque colony variant to the avirulent translucent colony variant ${ }^{15}$.

Rel, RelA and SpoT all share the same conserved domain composition, indicative of a common architecture of the underlying intra-molecular allosteric regulation in long RSHs ${ }^{1}$. When recruited to starved ribosomes, both Rel and RelA adopt a highly extended elongated 
conformation. In these complexes the regulatory C-terminal domain region (CTD: TGS, HEL, ZFD and RRM domains) is highly structured, while the N-terminal catalytic region (NTD: HD and SYNTH domains) and the interdomain linker regions are highly dynamic and unresolved in some structures ${ }^{18-21}$. Off the ribosome, our structural understanding of long RSHs relies on the structures of isolated NTDs of several Rel representatives ${ }^{21-24}$. While the physiological role of SpoT as a key virulence and stress tolerance factor is well established ${ }^{25,26}$, structural insights into SpoT are lacking altogether. This limits our ability to interpret the physiological and microbiological studies on the molecular level. Obtaining full-length structures of Rel/RelA/SpoT is essential for understating how the auto-regulation signal transferred from the CTD to the NTD.

The structural and biochemical data presented here provide the long-missing structural insight into the molecular mechanism of SpoT. We show that $A$. baumannii SpoT $\left(\mathrm{SpoT}_{A b}\right)$ is indeed, a monofunctional (p)ppGpp hydrolase and uncover how its CTD is an allosteric activator of the HD hydrolase function. The structures of the full-length HD-active SpoT $_{A b}$ complexed with the ppGpp substrate reveal a compact monomeric conformation in which all the regulatory domains wrap around a Core subdomain that connects the pseudoSYNTH and TGS domains. The Core is one of the intrinsically disordered regions (IDR) present in Rel and RelA when in the active synthetase state. In $\operatorname{SpoT}_{A b}$, Core and TGS cooperate to align and activate the hydrolase domain active site while translating allosteric feedback from the other regulatory domains to modulate the HD output. Finally, we propose a unifying conceptual framework that rationalises the relative balance between HD vs SYNTH activities of long RSHs Rel, RelA and SpoT, fine-tuned through the entropic force produced by intrinsically disordered regions that function as conformational gatekeepers of the enzyme. 


\section{A. baumannii $\operatorname{SpoT}_{A b}$ is a monofunctional hydrolase long RSH}

Lack of conservation of active site residues critical for SYNTH activity suggest that Moraxallaceae SpoT enzymes have - like RelA - undergone subfunctionalisation to become monofunctional long RSHs (Fig. 1a and b). Like RelA's pseudo-HD domain, the SYNTH domain region has been retained in Moraxallaceae as a presumably non-catalytic pseudoSYNTH domain, suggesting it retains some function in stabilisation or allosteric regulation of

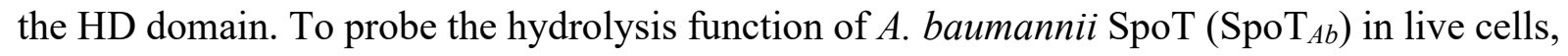
we leveraged SpoT's hydrolytic activity being crucial for controlling the cellular levels of (p)ppGpp produced by RelA, which makes spoT conditionally essential in the relA $A^{+}$ Escherichia coli ${ }^{12}$. We co-transformed a $\operatorname{ppGpp}^{0}(\Delta$ relA $\Delta$ spoT) E. coli strain with i) a pMG25based plasmid driving the IPTG-inducible expression of $s p o T_{\mathrm{Ab}}$ under the control of $\mathrm{P}_{\mathrm{A} 1 / \mathrm{O} 4 / \mathrm{O} 3}$ promoter and ii) a pMR33 derivative for arabinose-inducible expression of rel $A_{\mathrm{Ec}}$ under the control of $\mathrm{P}_{\mathrm{BAD}}$. While expression of the (p)ppGpp synthetase RelA $\mathrm{A}_{E c}$ strongly inhibited the growth of $\mathrm{ppGpp}^{0}$ E. coli, the growth was completely restored upon the ectopic co-expression of $\operatorname{SpoT}_{A b}$ (Fig. 1c and Supplemental Data), demonstrating that $\mathrm{SpoT}_{A b}$ is HD-active in the surrogate E. coli host.

Next, we used our dual plasmid co-expression system to probe the (p)ppGpp synthetase activity of SpoT RSHs. $\mathrm{pgGp}^{0}$ E. coli is auxotrophic for eleven amino acids, and (p)ppGpp synthetase activity of $\mathrm{SpoT}_{E c}$ is essential for growth of $\Delta$ relA E. coli on minimal medium ${ }^{12}$. Unlike the SYNTH-active $\operatorname{SpoT}_{E c}$, SpoT $_{A b}$ failed to promote the growth of $\mathrm{ppGpp}^{0}$ E. coli on M9 minimal medium (Fig. 1d), confirming that $\mathrm{SpoT}_{A b}$ is SYNTH-inactive. Taken together, these results demonstrate that $\mathrm{SpoT}_{A b}$ is a specialised monofunctional long RSH that lacks the ability to synthesise (p)ppGpp.

\section{Full-length $\operatorname{SpoT}_{A b}$ has a compact mushroom-like $\tau$-shaped structure}

To gain insight into the molecular workings of SpoT, we solved an X-ray structure of fulllength catalytically-active $\operatorname{SpoT}_{A b}$ in a ppGpp-bound state at $2.9 \AA$ resolution. The structure revealed a multi-domain architecture strikingly different to that observed earlier for ribosomebound long RSHs Rel and RelA ${ }^{18-21}$ (Fig. 2a-c and Supplementary Table 1). The HD, SYNTH, TGS, HEL, ZFD and RRM domains of $\operatorname{SpoT}_{A b}$ form a mushroom-like tau $(\tau)$-shaped quaternary structure (Fig. 2a-c). In this arrangement, pseudo-SYNTH, TGS, HEL, ZFD and RRM domains all lie in a single plane and form a compact disc-like structure that forms the “cap" of the "mushroom" (Fig. 2b). A helix-turn-helix sub-domain (residues 334 to 379) that 
provides the transition between the NTD and CTD regions, lies at the "Core" of the "cap" and seemingly mediates interactions among all domains of the enzyme. Such an arrangement suggests that the Core - which is disordered in Rel/RelA structures - stabilises the disc-like “cap" of SpoT (Fig. 2c). Moreover, the Core provides the HD domain with a physical link to each domain of $\operatorname{SpoT}_{A b}$. Finally, the HD protrudes from the plane of the "cap" in the opposite direction of the C-terminal RRM domain, forming the "stem" of the protein structure (Fig. 2bc).

The $\tau$-shaped structure of SpoT $_{A b}$ suggests a possible structural mechanism for the autoinhibition of SYNTH activity by the regulatory CTD both in Rel ${ }^{27,28}$ and RelA ${ }^{29,30}$. While the SYNTH and TGS domains are sequestered in the "cap", the HD hydrolase stands out unconfined and primed for (p)ppGpp hydrolysis. The TGS domain, which in the case of amino acid starvation sensors Rel and RelA specifically engages the deacylated tRNA CCA-3' end at the A site ${ }^{19-21,31}$, in the case of $\operatorname{SpoT}_{A b}$ is partially trapped between the HD, HEL and ZFD domains. While we do detect a mild inhibitory effect of tRNA on $\operatorname{SpoT}_{A b}$ hydrolysis activity, the effect is insensitive to tRNA aminoacylation status, i.e. non-specific (Fig. 2d). This is in contrast to the HD activity of bifunctional E. coli SpoT $\left(\operatorname{SpoT}_{E c}\right)$, which was specifically inhibited by deacylated, but not aminoacylated tRNA ${ }^{32}$.

Our structure reveals that the sites from the ZFD and RRM domains that mediate rRNA recognition in Rel/RelA ${ }^{18-21,31}$ are held in by the Core subdomain, suggesting that in the $\tau$ shaped conformation the hydrolytically active $\left(\mathrm{HD}^{\mathrm{ON}}\right) \operatorname{SpoT}_{A b}$ is incompatible with ribosome binding. In good agreement with this structural prediction, while the ribosome strongly suppresses the HD activity of Bacillus subtilis $\operatorname{Rel}\left(\operatorname{Rel}_{B s}\right)^{28}$, the addition of $E$. coli $70 \mathrm{~S}$ has no effect on the hydrolysis activity of $\operatorname{SpoT}_{A b}$ (Fig. 2d). Thus, our biochemical results suggest that $\mathrm{SpoT}_{A b}$ is a ribosome-independent enzyme.

Shorter intrinsically disordered regions (IDRs) in monofunctional SpoT are associated with specialisation for hydrolysis The presence of intrinsically disordered regions (IDR) located at the $\alpha 6-\alpha 7$ loop, the Core subdomain and the linker between HEL/ZFD domains in long RSHs RelA and Rel (Supplementary Fig. 1a) has posed an experimental challenge for structural studies ${ }^{18-21}$. The molecular function of these flexible regions, unresolved in the structures, is unknown. Comparison between the well-structured $\mathrm{SpoT}_{A b}$ in $\tau$-state and partially unstructured ribosomebound RelA/Rel suggests that the unfolding of Core and HEL domains constitutes part of the 
conformational switch that positions TGS, ZFD and RRM domains to stimulate the synthesis activity of Rel/RelA upon recruitment to the ribosome (Supplementary Fig. 1b).

The length of these disordered or flexible regions is on average shorter in monofunctional SpoT and much longer in the monofunctional RelA. Bifunctional Rels have interdomain IDRs of sizes between both monofunctional enzymes (Supplementary Fig. 1c). The $\alpha 6-\alpha 7$ loop of the HD domain of SpoT[Hs] in particular is a third of the size of that of RelA, which, in turn, is twice longer than that of bifunctional Rel (Supplementary Fig. 1c). The same pattern is observed for the other two IDRs: the Core subdomain and the region connecting HEL and ZFD domains. This is consistent with the significantly lower disordered propensity of the Core of $\mathrm{SpoT}_{A b}$ compared to RelA $A b$ (Supplementary Fig. 1d-e). We speculate that these IDRs have evolved to stabilise either $\tau$ - (shorter IDRs) or elongated (longer IDRs) states of monofunctional SpoT[Hs] or RelA[hS], respectively, to tune the HD vs SYNTH output ratio.

\section{$\operatorname{SpoT}_{A b}$ is a monomer}

It was shown earlier that both Rel and RelA are prone to dimerization via the CTD, which would potentially serve to regulate their enzymatic activity ${ }^{21,33-35}$. This idea is a subject of debate, with both genetic ${ }^{30}$ and mass photometry ${ }^{28}$ experiments suggesting that the dimerization is unlikely to take place at physiologically relevant concentrations. Therefore, we used small-angle X-ray scattering (SAXS) coupled to size exclusion chromatography (SEC) to probe the conformation and oligomeric state of $\mathrm{SpoT}_{A b}$ in solution (Fig. 2e-f).

The SAXS data revealed that in solution $\mathrm{SpoT}_{A b}$ has an oblate shape compatible with the structure determined by X-ray. Both SAXS and SEC consistently support the monomeric nature of $\operatorname{SpoT}_{A b}$, even at concentrations as high as $8 \mathrm{mg} / \mathrm{mL}$. Both the molecular weight of $\approx 90$ $\mathrm{kDa}$ by SEC as well the estimates of $\mathrm{Mw}$ of $\approx 85 \mathrm{kDa}$ and $R \mathrm{~g}$ of $34.9 \AA$ by SAXS (Fig. 2e-f) agree with the $80 \mathrm{kDa}$ theoretical molecular weight of monomeric $\mathrm{SpoT}_{A b}$. Furthermore, the analysis of the normalised Kratky plot derived from the scattering curve lends further support for a compact monomeric structure of $\mathrm{SpoT}_{A b}$ in solution (Fig. 2f), and the ab initio envelope calculated from the experimental SAXS data (Fig. 2g) is compatible with the $\tau$-shaped structure of $\mathrm{SpoT}_{A b}$ determined by X-ray. Collectively these results demonstrate that in solution the monomeric $\operatorname{SpoT}_{A b}$ adopts a conformation that is very similar to the $\tau$-shaped conformation observed in the crystal with the HD domain protruding from the disc-shaped enzyme.

\section{The enzymatically-inactive pseudo-SYNTH of $\operatorname{SpoT}_{A b}$ is a regulatory domain}


In the monofunctional stringent factor RelA, the enzymatically inactive pseudo-HD domain has evolved into a regulatory domain controlling catalysis via an intra-NTD allosteric regulatory mechanism ${ }^{36,37}$. This is also the case with the specialisation of $\operatorname{SpoT}_{A b}$ as a monofunctional hydrolase where the pseudo-SYNTH domain has evolved into a strictly regulatory/structural domain. Superposition of the SYNTH domain from $\operatorname{Rel}_{T t}$ onto the pseudo-SYNTH domain of $\mathrm{SpoT}_{A b}$ reveals extensive reorganisation of the vestigial catalytic domain in $\mathrm{SpoT}_{A b}$, consistent with differential conservation patterns in the G-loop and the ATP recognition motif (Supplementary Fig. 2a). These involve the residues that coordinate adenosine and guanosine (R249 to N241, R277 to E267 and Y329 to N304) and the majority of phosphate-coordinating groups. Crucially, the catalytic residues D272 and Q347 are substituted for S263 and T321, respectively. These substitutions essentially impede the deprotonation and activation of the 3'$\mathrm{OH}$ of $\mathrm{GD}(\mathrm{T}) \mathrm{P}$, and $\mathrm{Mg}^{2+}$ binding, precluding the nucleophilic attack on the $\beta$-phosphate of ATP. We directly probed GDP binding by $\operatorname{SpoT}_{A b}{ }^{\mathrm{NTD}}$ and RelA ${ }_{A b}{ }^{\mathrm{NTD}}$ by ITC. As expected, while $\operatorname{SpoT}_{A b}$ does not bind GDP, RelA $A b$ binds GDP with an affinity of $62 \mu \mathrm{M}$, which is similar

\section{$\operatorname{SpoT}_{A b}$ is not allosterically regulated by the alarmone pppGpp}

The enzymatic activity of long RSHs is regulated via strong allosteric coupling between the HD and SYNTH domains that results in antagonistic conformational states ${ }^{22,23,36}$. While in Rel/RelA (p)ppGpp bind the hinge region connecting the SYNTH and HD/pseudo-HD domains to stimulate the SYNTH activity, this regulation is lost in $\operatorname{SpoT}_{E c}{ }^{36}$. Our structure of $\operatorname{SpoT}_{A b}$ provides a mechanistic interpretation. In the $\tau$-state the highly structured Core subdomain makes numerous contacts with SYNTH providing further scaffolding to the already more stable version of the HD:SYNTH hinge of $\operatorname{SpoT}_{A b}$. Additionally, there are several important substitutions in the (p)ppGpp binding site that would be expected to compromise (p)ppGpp binding and alarmone-mediated regulation, specifically in Q203 (a residue involved in ribose coordination and strictly conserved as A in RelA ${ }^{36}$ ) and in T209 (a residue involved in phosphate coordination, typically $\mathrm{K}$ or R in RelA ${ }^{36}$ ).

To directly validate the lack of pppGpp-mediated regulation in $\mathrm{SpoT}_{A b}$, we characterised the interaction between pppGpp and $\mathrm{SpoT}_{A b}{ }^{\mathrm{NTD}}$ by ITC. As expected, $\mathrm{SpoT}_{A b}{ }^{\mathrm{NTD}}$ does not bind pppGpp allosterically (Supplementary Fig. 2d-e). Following the experimental approach used earlier for $\operatorname{SpoT}_{E c}{ }^{36}$, we next grafted the allosteric site of $A$. baumannii $\operatorname{RelA}\left({ }^{236} \operatorname{RelA}^{246}\right)$ onto $\mathrm{SpoT}_{A b}{ }^{\mathrm{NTD}}$ (replacing ${ }^{201} \mathrm{SpoT}_{A b}{ }^{211}$ ). Just as in the case of $\mathrm{SpoT}_{E c}$, this resulted in a RelA-like affinity to pppGpp of the chimera $\mathrm{RSH}\left(K_{D}=5.6 \mu \mathrm{M}\right)$. Collectively, these results support the 
generality of alarmone-mediated control being lost in SpoT and only present in SYNTH-active Rel/RelA stringent factors that mediate acute stringent response upon amino acid starvation.

\section{The dipolar architecture of the HD active site is conserved between Rel and SpoT}

244 Inspection of the electron density map of the $\mathrm{SpoT}_{A b}$-ppGpp complex reveals that the alarmone is bound in high occupancy in each of the four $\mathrm{SpoT}_{A b}$ molecules present in the asymmetric unit of the crystal (Supplementary Fig. 2f), with the coordination of the guanine base of ppGpp (Fig. 3a-c) resembling that observed in $\operatorname{Rel}_{T t}{ }^{\mathrm{NTD}}-$ ppGpp ${ }^{23}$ and $\mathrm{Rel}_{T t}{ }_{{ }^{\mathrm{NTD}}}$-pppGpp complexes ${ }^{24}$. We probed enzymatically the role of each residue involved in guanine coordination via systematic Ala-substitutions. While substitution of R45 (stacking the guanine) abrogated hydrolysis, removing Van der Waals contacts to L154 decreased the activity approximately two-fold; interactions with K46 were redundant (Fig. 3d). Disruption of the hydrogen bond of the guanine to T150 had only a minor effect. The additional hydrogen bond formed between the carbonyl group of the guanine and the enzyme's backbone likely accounts for the guanine specificity of SpoT over adenosine.

As observed earlier for $\operatorname{Rel}_{T t}{ }^{\mathrm{NTD}} 23$, the hydrolase active site of $\mathrm{SpoT}_{A b}$ displays a dipolar charge distribution with a highly basic half mediating the stabilization of the 5'- and 3'polyphosphate groups of the substrate and the other highly acidic half mediating the 3 'pyrophosphate hydrolysis (Fig. 3a-b). Closer inspection of the complex reveals the crucial role of Y51 and the ${ }^{82} \mathrm{ED}^{83}$ active site motifs as they work together with the $\mathrm{Mn}^{2+}$ cofactor to coordinate and stabilise a network of water molecules near the sugar-phosphate moiety during hydrolysis (Fig. 3b-c). Indeed, substitutions of Y51, E82, D83 or N147 render SpoT $A b$ HDinactive in our enzymatic assays (Fig. 3d). At the positively charged side active site the 5'polyphosphate is loosely coordinated and exposed to the bulk solvent. By contrast K140 and R144 hold the 3'-pyrophosphate in place during hydrolysis and Ala substitutions of these residues decrease the activity of the enzyme between 5- and 10-fold suggesting these are key residues that orient the scissile bond.

\section{$\mathrm{Mn}^{2+}$ ion organizes the HD active site of $\operatorname{SpoT}_{A b}$}

The essential role of the divalent manganese ion $\mathrm{Mn}^{2+}$ in (p)ppGpp pyrophosphate hydrolysis (ITC) measurements demonstrate that unliganded, metal-free $\mathrm{SpoT}_{A b}{ }^{\mathrm{NTD}}$ binds $\mathrm{Mn}^{2+}$ with a $K_{\mathrm{D}}$ of $35.3 \mu \mathrm{M}$ (Fig. 3e). Furthermore, while metal-free full-length $\mathrm{SpoT}_{A b}$ is completely HDinactive, the HD activity is readily restored upon addition of $\mathrm{Mn}^{2+}$ (Fig. 3f). 
To directly reveal the structural role of $\mathrm{Mn}^{2+}$ we determined the X-ray structure of SpoT $_{A b}{ }^{\mathrm{NTD}}$ in the metal-free state (Fig. 3g and Supplementary Table 1). Comparison with the structure of the $\mathrm{SpoT}_{A b}$-ppGpp complex provides a structural explanation for the essentiality of $\mathrm{Mn}^{2+}$ for catalysis: in addition to its role in hydrolysis, by connecting $\alpha 3, \alpha 4$ and $\alpha 8, \mathrm{Mn}^{2+}$ coordination brings together the two halves of the HD domain and provides structural support to the active site (Fig. 3g-h). While the overall topology of the $\operatorname{SpoT}_{A b}$ HD domain is similar to that of $\mathrm{Mn}^{2+}$-liganded $\operatorname{Rel}_{T t}{ }^{\mathrm{NTD}}{ }^{23}$, the removal of the metal ion has a profound effect on the local conformation of the active site of $\mathrm{SpoT}_{A b}{ }^{\mathrm{NTD}}$. The catalytic ${ }^{78} \mathrm{HD}^{79}$ and ${ }^{82} \mathrm{ED}^{83}$ motifs are largely misaligned, loops S110-Y117 and A153-K158 that are involved in the 3'- and 5'phosphate coordination are disordered, and the guanine-coordinating loop T44-Y51 assumes a conformation incompatible with the base coordination (Fig. 3h). Importantly, all of these changes do not result in the opening of the enzyme's NTD that was observed in $\mathrm{Rel}_{T t}$ upon removal of $\mathrm{Mn}^{2+23}$. These observations suggest that with the evolution as a monofunctional enzyme, $\operatorname{SpoT}_{A b}$ shed the allosteric conformational control between the HD and pseudoSYNTH domains.

\section{The CTD allosterically stimulates the hydrolysis activity of the SpoT NTD}

Until now, our understanding of the function of the CTD region of long RSHs was based exclusively on studies of Rel and RelA. This has established a role of the CTD in the association of the stringent factors with starved ribosomes resulting in the activation of the SYNTH activity and the auto-inhibition of the factor's SYNTH activity off the ribosome 18-21,27,28. Weak hydrolase activity of the CTD-truncated Rel has also indicated a possible HD-stimulatory role of the CTD through an intra-molecular regulation of the hydrolase function ${ }^{28,41,42}$, suggesting that a similar mechanism could also be at play in the case of SpoT.

To probe this hypothesis, we characterised the HD activity - both in vitro and in vivo of a set of progressively C-terminally truncated variants of $\operatorname{SpoT}_{A b}$ lacking i) RRM (SpoT ${ }_{A b}{ }^{1-}$ ${ }^{614}$, amino acids 1-614), ii) RRM and ZFD ( $\left.\operatorname{SpoT}_{A b}{ }^{1-560}\right)$, iii) RRM, ZFD and HEL (SpoT ${ }_{A b}{ }^{1-}$ $\left.{ }^{454}\right)$, iv) CTD altogether, i.e. RRM, ZFD, HEL and TGS $\left(\operatorname{SpoT}_{A b}{ }^{1-385}\right)$, v) CTD as well as the Core domain $\left(\operatorname{SpoT}_{A b^{1-339}}\right)$, and finally, a variant that consisted of just the HD domain $\left(\operatorname{SpoT}_{A b^{1-}}\right.$ ${ }^{195}$ ). These truncated variants were all generated at the endogenous spoT locus in a $\Delta$ relA Ptac::relA A. baumannii strain, and the ability to grow on complex media supplemented with IPTG was evaluated as a proxy of the (p)ppGpp hydrolase activity of $\operatorname{SpoT}_{A b}$ in vivo.

While $\mathrm{SpoT}_{A b}$ variants lacking the RRM or the RRM and ZFD domains retained wildtype ability to sustain the bacterial growth grow - i.e. could efficiently degrade (p)ppGpp 
synthesised by RelA - further C-terminal truncations compromised the in vivo $\mathrm{HD}$

309 functionality, as evidenced from pronounced growth defects (Fig. 4a). Biochemical assays are 310 in agreement with the in vivo data (Fig. $\mathbf{4 b}$ ). Truncation of the RRM and ZFD decreases the 311 HD activity 5-fold. Further deletion of the TGS-HEL domains leads to a dramatic 42-fold 312 decrease in activity. Truncations beyond the TGS compromised the activity by 70 -fold or more 313 and isolated HD domain was nearly inactive. Collectively, our results suggest that the CTD 314 region functions as an allosteric activator of the hydrolase function of $\mathrm{SpoT}_{A b}$. Next, we set out 315 to dissect the molecular mechanism of the CTD-mediated NTD control and assign the 316 molecular functions to individual CTD domains.

\section{The Core domain is a linchpin that controls the $\tau$-state}

319 Both the overall structural arrangement of $\operatorname{SpoT}_{A b}$ and our sequential domain truncation 320 experiments (Fig. 4a-b) suggest that the Core-mediated allosteric crosstalk between the HD 321 and rest of the domains of the enzyme is essential for enzyme's functionality. To specifically 322 assess the role of the individual interdomain interactions we introduced single point substitutions at each of the interfaces of the Core with regulatory CTD domains (Fig. $\mathbf{4 c}$ ) and measured the hydrolase activity of the $\mathrm{SpoT}_{A b}$ variants (Fig. 4d). An intact HD:Core:TGS interface - the structure involved in scaffolding the HD active site - is crucial for HD activity, as the Y375G substitution at the HD:Core:TGS resulted in a 5-fold decrease in activity compared to the wild type. While substitutions at the ZFD (L373G / D374G) and RRM $(\mathrm{A} 351 \mathrm{~K})$ domain interfaces also resulted in a pronounced defect (19 and 3-fold decrease, respectively), perturbations at the Core:pseudo-SYNTH domain interface (A348R) had only a minor effect on hydrolysis. Finally, decoupling the contacts of HD from the $\tau$-cap via the L356D substitution, located at the interface between Core domain and $\alpha 6-\alpha 7$ motif of HD ${ }^{23}$, has a dramatic 35-fold decrease in HD activity, suggestive of an allosteric signal transduction path 333 between the cap and stem regions of the enzyme. When we monitored the thermodynamic stability of these Core variants of $\operatorname{SpoT}_{A b}$ we observed they all have lower stability and loss of structure compared to the wild type (Supplementary Fig. 3a-e). This suggests that an increase in the configurational entropy of the Core has a global effect in the dynamics and compactness of the enzyme. The existence of an allosteric relay mediating a CTD-dependent activation of 338 HD via the Core is further supported by the consistent decrease in hydrolysis associated with the aforementioned C-terminal truncations that affect the feedback of the Core to the HD (Fig. 4a-b), as well as by the observation that the deletion of domains HEL and TGS results in a 50- 
fold decrease in activity despite the presence of the other regulatory domains (pseudo-SYNTH, ZFD and RRM) (Fig. 4d).

We next used SEC-SAXS to directly probe the role of each contact at the interface of the Core with the different domains of $\operatorname{SpoT}_{A b}$ on stabilisation of the $\tau$-sate. The L356D substitution ( $\mathrm{SpoT}_{A b}{ }^{\mathrm{L} 356 \mathrm{D}}$, Fig. $4 \mathbf{c}$ and Supplementary Table 2) results in the segregation of the population into two conformational states with major differences in $R_{G}$ (radius of gyration) and particle dimensions $\left(D_{M A X}\right)$. In $\operatorname{SpoT}_{A b}{ }^{\mathrm{L} 356 \mathrm{D}}$ one state is the compact $\tau$-shape observed in the crystal structure (Fig. 4e), while the other state is more relaxed $\left(R_{G}=41 \AA, D_{M A X}=130 \AA\right)$ with dimensions reminiscent of that of the less compacted Rel and RelA - but not quite as elongated as in the ribosome-bound state (Fig. 4f). In this relaxed state the Core and HEL domains appear to have transitioned to a more disordered state that is consistent with the conformational states of these regions in the fully elongated state observed in Rel/RelA (Fig. 4g-h); the other domains retain their structural integrity. Prompted by this analogy, we next probed A. baumannii monofunctional synthetase RelA and B. subtilis bifunctional RSH Rel $\mathrm{B}_{B S}$ with SAXS. The dimensions of $\operatorname{RelA}_{A b}\left(R_{G}=42 \AA, D_{M A X}=130 \AA, \mathrm{Mw}=88 \mathrm{kDa}\right)$ are consistent with that of the relaxed state of $\mathrm{SpoT}_{A b}{ }^{\mathrm{L} 356 \mathrm{D}}$, whereas $\mathrm{Rel}_{B S}$ is populated by both the relaxed and $\tau$-states (Fig. 4i-k and Supplementary Table 2).

Collectively, our results suggest that the Core domain functions as an allosteric relay that conveys signals from the CTD to the HD. At the structural level the composition of the Core is the key to the conformational state of the enzyme as defined by the three major conformations observed in SpoT, Rel and RelA (Fig. 41). The correlation of the decrease in HD activity with entropy-increasing substitutions such as A351K, L356D, L371G / D374G, and Y375G supports the notion that the increase in structural disorder or flexibility of the Core domain (or the other IDRs) likely drives the conformational equilibrium of the enzyme away from the $\tau$-state. This decrease in activity observed by the disruption of the $\tau$-shape is also consistent with the lack of hydrolysis in Rel homologues that underwent an order-to-disordered transition while accommodating in ribosomal A site ${ }^{28}$. In this context the aforementioned relaxed state is likely the idle resting state of long RSH enzymes in which the CTD precludes the function of SYNTH while not activating HD.

\section{The TGS domain acts as a scaffold for the HD active site}

The $\alpha 6-\alpha 7$ element plays a crucial role in the allosteric regulation of the opposing activities of bifunctional $\operatorname{Rel}_{T t}{ }^{23}$. In $\operatorname{Rel}_{T t}, \alpha 6-\alpha 7$ of projects away from the HD catalytic centre to accommodate the $3^{\prime}$ and $5^{\prime}$ polyphosphate groups as well as allowing the catalytic ${ }^{82} \mathrm{ED}^{83}$ motif 
to get in position, close to the $3^{\prime}$ phosphates, priming the enzyme for hydrolysis. In $\mathrm{SpoT}_{A b}$ the outward-pointing conformation of $\alpha 6-\alpha 7$ is further stabilised by the N-terminal region of the TGS and the Core domains which function as a clamp to keep $\alpha 6-\alpha 7$ in the HD-compatible position, with the HEL domain providing an additional support via the Core (Fig. 5a). The dramatic drop in the activity of the $\operatorname{SpoT}_{A b}$ variant lacking the TGS and HEL domains (Fig. 4d) substantiates the functional importance of this stabilising effect.

At the HD:TGS interface the $\beta$-hairpin of the TGS - the very element which is involved in tRNA recognition in Rel ${ }^{21,28,42}$ and RelA ${ }^{19,20,31}$ - is buried and stacking directly the $\alpha 6-\alpha 7$ element via a small hydrophobic interface formed by W382, Y384, L390 and the R124-E392 salt bridge (Fig. 5a). Disrupting this interface with the E379K / W382K substitutions $\left(\operatorname{SpoT}_{A b}{ }^{\mathrm{E} 379 \mathrm{~K} / \mathrm{W} 382 \mathrm{~K}}\right)$ led to a 17 -fold decrease in the hydrolase activity of the enzyme (Fig. 4d) suggesting that the HD:TGS interface constitutes as an important allosteric signal transduction pathway. This scaffolding role is complemented by the Core that wraps tightly around $\alpha 7$ thus preventing the recoil of $\alpha 6-\alpha 7$ away of the HD active site, which, as we observed earlier in Rel $\mathrm{l}_{T t}$ ${ }^{23}$, induces the opening of the NTD . Indeed, substitutions at the Core: $\alpha 6-\alpha 7$ interface such as the aforementioned Y375G also affected hydrolysis (Fig. 4c-d). Interestingly, despite the strongly attenuated HD activity of $\mathrm{SpoT}_{A b}{ }^{\mathrm{E} 379 \mathrm{~K} / \mathrm{W} 382 \mathrm{~K}}$, SAXS showed $\mathrm{SpoT}_{A b}{ }^{\mathrm{E} 379 \mathrm{~K} / \mathrm{W} 382 \mathrm{~K}}$ remains in the $\tau$-state $\left(R_{G}=35 \AA, D_{M A X}=104 \AA\right)$, suggesting an allosteric communication via the HD:Core:TGS axis (Fig. 5b and Supplementary Table 2).

Given that $\mathrm{SpoT}_{A b}$ is SYNTH-inactive and is not specifically regulated by tRNA or ribosomes (Fig. 2d), it is not surprising that TGS residues involved in tRNA recognition - such as the crucial His residue involved in the recognition of the 3' CCA end by Rel ${ }^{21,28,42}$ and RelA ${ }^{19,31}\left(\mathrm{~S} 407\right.$ in $\left.\operatorname{SpoT}_{A b}\right)$ - are lost in the monofunctional $\operatorname{SpoT}_{A b}$ (but are present in bifunctional $\left.\operatorname{SpoT}_{E c}{ }^{1}\right)$. Moreover, the $\tau$-state is sterically incompatible with the potential recognition of tRNA by TGS due to sequestration the $\beta$-hairpin and $\alpha$-helical elements. All these observations suggest that in $\operatorname{SpoT}_{A b}$ the TGS has been repurposed as a scaffolding domain crucial to sustain hydrolysis, with both TGS and Core cooperating to lock the $\alpha 6-\alpha 7$ in place, stabilising the HD active site. This contrasts with its crucial function of recognition of uncharged tRNA in Rel/RelA ${ }^{19-21,28,31}$.

\section{The ZFD and RRM domains finetune the hydrolytic activity of $\operatorname{SpoT}_{A b}$}

With ZFD and RRM positioned close to the disc-shaped cap and connecting with the pseudoSYNTH domain, the resulting inter-domain interfaces are likely to play a role in the stability the $\tau$-state as well as to allosterically control of HD via the HD:pseudo-SYNTH relay. In 
agreement with this hypothesis, disruptive substitutions at the Core:HD (L356D), Core:pseudo-

410 SYNTH:RRM (A351K) and Core:ZFD (L373G / D374G) that decreased the stability of the $\tau$ 411 state (Supplementary Fig. 3b-e) also decreased the HD activity of the enzyme by 35-, 3- and 412 22-fold, respectively (Fig. 4d). Therefore, we reasoned that substitutions stabilising the 413 Core:pseudo-SYNTH:RRM and Core:ZFD interfaces would, conversely, trigger an allosteric 414 activation of hydrolysis.

To probe this hypothesis, we introduced substitutions that would increase the contacts of RRM with pseudo-SYNTH via hydrogen bonds, I637D / R641D, and the Core with the ZDF, D374R (Fig. 5c). Denaturation experiments showed $\mathrm{SpoT}_{A b}{ }^{\mathrm{D} 374 \mathrm{R}}$ and $\mathrm{SpoT}_{A b}{ }^{\mathrm{I} 637 \mathrm{D} / \mathrm{R} 641 \mathrm{D}}$ have higher stability and compactness than the WT (Supplementary Fig 4a-c) and SAXS measurements on $\operatorname{SpoT}_{A b}{ }^{1637 \mathrm{D} / \mathrm{R} 641 \mathrm{D}}$ confirmed this variant retained the $\tau$-state $($ Fig. $5 \mathbf{d}$ and Supplementary Table 2). As expected, the HD turnover of both enzyme variants increased (by 2.1- and 1.6-fold, respectively, Fig. 4d), and both behave like wild-type $\mathrm{SpoT}_{A b}$ in vivo (Fig. 5e).

Collectively, our results establish that HD activity is coupled to the stability of the $\tau$ state, with the Core domain working as an allosteric transducer that allows the catalytic HD to communicate with all the regulatory domains. Substitutions or interactions that stabilise the $\tau$ state increase hydrolysis, whereas $\tau$-state-destabilising substitutions lower the HD activity.

\section{An intact $\tau$-shaped $\operatorname{SpoT}_{A b}$ is required for virulence of $A$. baumannii}

Functional (p)ppGpp-mediated signalling plays a crucial role in antibiotic tolerance and virulence of $A$. baumannii ${ }^{15,43}$. We used the wax moth $G$. mellonella larvae infection model to assess the functionality of mutant $s p o T_{\mathrm{Ab}}$ variants in supporting virulence of $A$. baumannii AB5075 (Fig. 5f). Only the strain with wild type-like virulence was the one expressing $\operatorname{SpoT}_{A b}$ D374R variant with a HD activity slightly higher than that of the WT SpoT. The $s p o T_{\mathrm{Ab}}{ }^{\mathrm{D} 374 \mathrm{R}}$ strain has rapidly killed $100 \%$ of the larvae within the first two days whereas $60 \%$ of the larvae survived 6 days of infection with the (p)ppGpp ${ }^{0} \Delta r e l A$ strain. Infection with A. baumannii expressing the $\triangle \mathrm{RRM}$-truncated enzyme $\operatorname{SpoT}_{A b}{ }^{1-614}$ resulted in $25 \%$ survival rate of larvae after 6 days. Notably, the RRM-truncated $\operatorname{SpoT}_{A b}{ }^{1-614}$ had 6-fold lower hydrolase activity as compared to wild type (Fig. 4d), and the strain displays no growth defects when grown on LB plates (Fig. 4a). The defect in virulence becomes more prominent with truncations beyond the TGS domain: $\operatorname{SpoT}_{A b^{1-454}}$ and $\mathrm{SpoT}_{A b}{ }^{1-339}$. The strong decrease in HD activity associated with the A. baumannii strains expressing these SpoT variants results in $100 \%$ survival of the infected larvae (Fig. 5f). Collectively our results suggest that while a basal level of the HD hydrolase 
activity is sufficient to sustain bacterial growth in non-stressed conditions (e.g. on a plate and in liquid culture), the pathogen requires fully functional CTD- and Core-mediated control of $\operatorname{SpoT}_{A b}$ to tune the HD activity and efficiently establish a successful infection.

\section{Discussion}

This study reveals the unexpected $\tau$-shaped architecture of full-length monofunctional $\operatorname{SpoT}_{A b}$, which enables auto-stimulation of the hydrolase activity of the enzyme by its CTD. With the loss of the synthetase function, the pseudo-SYNTH domain of $\operatorname{SpoT}_{A b}$ becomes a regulatory and structure stabilising domain. Together with TGS, HEL, ZFD and RRM, pseudo-SYNTH defines the interaction network that transmits the allosteric signal from the CTD to the HD active site via the Core of the enzyme, to regulate (p)ppGpp hydrolysis. The Core element, together with the TGS and $\mathrm{Mn}^{2+}$, aligns the active site residues of the HD in the correct position for catalysis. Compromising the functionality of either of these elements through substitutions of key residues results in major defects in hydrolysis activity. By contrast, pseudo-SYNTH, ZFD and RRM all subtly tune the HD activity of $\mathrm{SpoT}_{A b}$ up or down by modulating its interactions with the Core. Interestingly, the ribosome-associated Rel/RelA (p)ppGpp synthetases, lacking the Core are non-functional in vivo and SYNTH-inactive, with the minimal enzyme version with SYNTH activity consisting of HD/pseudo-HD, SYNTH and Core domains ${ }^{22,28,36,41}$. Therefore, the presence of the Core and its crosstalk with the HD/pseudo-HD domain likely constitutes universal structural requirement for the efficient stabilization of the active states of long RSH enzymes.

We propose a unifying scheme that rationalises the evolution of the enzymatic output of long RSHs through fine-tuning of the conformational equilibrium of the $\tau$, relaxed and ribosome-bound states of these enzyme (Fig. 6). The very presence of catalytically-competent synthetase and hydrolase domains in bifunctional Rel[HS] and SpoT[HS] requires both the $\tau$ and relaxed states as part of the conformational spectrum of these enzymes (Fig. 6a-b). While the $\tau$-state primes Rel/SpoT for efficient (p)ppGpp hydrolysis, the more elongated relaxed state sets the enzyme for low-efficiency (p)ppGpp synthesis. To fully activate its SYNTH activity, the enzyme needs to be further stimulated by starved ribosomes to attain the highly elongated ribosome-bound state; this transition is possible for amino acid starvation sensor Rel[HS], but not for SpoT, which is not under allosteric control by starved ribosomes and ppGpp ${ }^{36}$ (Fig. 6a). In the further subfunctionalised enzymes (dedicated hydrolase Moraxellaceae SpoT[Hs] and dedicated synthetase RelA[hS]) the intrinsic structural equilibrium is limited to a subset of the conformations accessible to ancestral bifunctional Rel[HS] (Fig. 6c-d). Compared to 
SpoT[HS], in SpoT[Hs] the equilibrium is further shifted towards the HD-active $\tau$-state required for hydrolysis (Fig. 6c). In contrast, in RelA[hS] the $\tau$-state becomes inaccessible, and the enzyme is primed for ribosomal recruitment upon which it is stabilised in the highly elongated ribosome-bound SYNTH-active state (Fig. 6d).

Expansion/contraction of the disordered regions is the likely molecular driver of the fine-tuning of the enzymatic output in long RSHs through the restriction of the conformational space (Supplementary Fig. 1c-e). Longer IDRs favour the relaxed state in RelA[hS] and increase the frustration of the enzyme, whereas the shorter IDRs favour the compact HD-active $\tau$-state in SpoT[Hs]. This genetic finetuning of a catalytic function, based on the optimization of the length and the forces generated by intrinsically disordered regions, is reminiscent of the evolution of human glucocorticoid receptor isoforms 44 or the UDP- $\alpha$-d-glucose-6dehydrogenase ${ }^{45}$. Such mechanisms seem to have evolved as a solution for conformationally heterogenous proteins with partially active resting states, that are under strong energetic and functional frustration.

The unifying scheme presented here provides a framework that can be used to rationalise the "hub" nature of SpoT and how binding partners such as the Acyl Carrier Protein (ACP) and the Regulator of RpoD $-\sigma^{70}$ - (Rsd) could modulate its output ${ }^{46,47}$ or in the case of Rel/RelA how the ribosome prevents hydrolysis by exploiting this extensive allosteric network. Other protein partners of Rel such EIIA ${ }^{\mathrm{NTR}}$ and DarB ${ }^{41,48}$ could also modulate the intramolecular allosteric communication of the regulatory domains with HD by favouring of the $\tau$ - or relaxed states, thus conditioning the catalytic output of the enzyme.

\section{Acknowledgments}

This work was supported by the Fonds National de Recherche Scientifique (FRFS-WELBIO CR-2017S-03, FNRS CDR J.0068.19, FNRS-EQP UN.025.19 and FNRS-PDR T.0066.18 to AGP); ERC (CoG DiStRes, $\mathrm{n}^{\circ} 864311$ to AGP) and Joint Programming Initiative on Antimicrobial Resistance, (JPIAMR) JPI-EC-AMR-R.8004.18 to AGP; the Program Actions de Recherche Concerté 2016-2021, Fonds d'Encouragement à la Recherche of ULB (AGP); Fonds Jean Brachet and the Fondation Van Buuren (AGP); Chargé de Recherches fellowship from the FNRS ${ }^{\circ}$ CR/DM-392 (HeT); the European Union's Horizon 2020 research and innovation programme under the Marie Skłodowska-Curie grant agreement No 801505 (IF@ULB postdoctoral grant to AA) and (FRFS-WELBIO-CR-2019S-05 to RH). AP is an F.R.S. - FNRS Postdoctoral Researcher and RH is an F.R.S. - FNRS Research Associate. We are also grateful the Protein Expertise Platform at Umeå University for constructing plasmids. 
511 This work was supported by the European Regional Development Fund through the Centre of 512 Excellence for Molecular Cell Technology (VH); project grant from the Knut and Alice 513 Wallenberg Foundation (2020-0037 to GCA); Ragnar Söderberg foundation (VH); Swedish 514 Research council (2019-01085 to GCA, 2017-03783 and 2021-01146 to VH, and 2018-00956

515 to VH under the framework of Joint Programming Initiative on Antimicrobial Resistance, 516 JPIAMR); the MIMS Excellence by Choice Postdoctoral Fellowship Programme grant 2018 517 (MR). The authors acknowledge the use of beamtimes PROXIMA 1 and 2A and SWING at the 518 Soleil synchrotron (Gif-sur-Yvette, France).

\section{Author contributions}

$521 \mathrm{VH}$ and AGP drafted the manuscript with contributions from all authors. AGP and VH 522 coordinated the study. VH, AGP, RH, HeT and MR designed experiments and analysed the 523 data. MR, HiT, SZ, JP, AT, HeT, PK, AT, AP, HA, AA performed experiments. RD, GL and 524 GCA performed bioinformatic analyses.

\section{Declaration of interests}

527 The authors declare no competing interests 


\section{References}

529 1. Atkinson, G.C., Tenson, T. \& Hauryliuk, V. The RelA/SpoT homolog (RSH)

530 superfamily: distribution and functional evolution of ppGpp synthetases and hydrolases across the tree of life. PLoS One 6, e23479 (2011).

533

2. Hauryliuk, V., Atkinson, G.C., Murakami, K.S., Tenson, T. \& Gerdes, K. Recent functional insights into the role of (p)ppGpp in bacterial physiology. Nat Rev Microbiol 13, 298-309 (2015).

3. Irving, S.E. \& Corrigan, R.M. Triggering the stringent response: signals responsible for activating (p)ppGpp synthesis in bacteria. Microbiology 164, 268-276 (2018).

4. Ronneau, S. \& Hallez, R. Make and break the alarmone: regulation of (p)ppGpp synthetase/hydrolase enzymes in bacteria. FEMS Microbiol Rev (2019).

5. Anderson, B.W., Fung, D.K. \& Wang, J.D. Regulatory Themes and Variations by the Stress-Signaling Nucleotide Alarmones (p)ppGpp in Bacteria. Annu Rev Genet (2021).

6. Steinchen, W., Zegarra, V. \& Bange, G. (p)ppGpp: Magic Modulators of Bacterial Physiology and Metabolism. Front Microbiol 11, 2072 (2020).

7. Haseltine, W.A. \& Block, R. Synthesis of guanosine tetra- and pentaphosphate requires the presence of a codon-specific, uncharged transfer ribonucleic acid in the acceptor site of ribosomes. Proc Natl Acad Sci U S A 70, 1564-8 (1973).

8. Pedersen, F.S. \& Kjeldgaard, N.O. Analysis of the relA gene product of Escherichia coli. Eur J Biochem 76, 91-7 (1977).

9. Shyp, V. et al. Positive allosteric feedback regulation of the stringent response enzyme RelA by its product. EMBO Rep 13, 835-9 (2012).

10. An, G., Justesen, J., Watson, R.J. \& Friesen, J.D. Cloning the spoT gene of Escherichia coli: identification of the spoT gene product. J Bacteriol 137, 1100-10 (1979).

11. Laffler, T. \& Gallant, J.A. Stringent control of protein synthesis in E. coli. Cell 3, 47-9 (1974).

12. Xiao, H. et al. Residual guanosine 3',5'-bispyrophosphate synthetic activity of relA null mutants can be eliminated by spoT null mutations. J Biol Chem 266, 5980-90 (1991).

13. Mittenhuber, G. Comparative genomics and evolution of genes encoding bacterial (p)ppGpp synthetases/hydrolases (the Rel, RelA and SpoT proteins). J Mol Microbiol Biotechnol 3, 585-600 (2001).

14. Jung, H.W., Kim, K., Islam, M.M., Lee, J.C. \& Shin, M. Role of ppGpp-regulated efflux genes in Acinetobacter baumannii. J Antimicrob Chemother 75, 1130-1134 (2020).

15. Perez-Varela, M., Tierney, A.R.P., Kim, J.S., Vazquez-Torres, A. \& Rather, P. Characterization of RelA in Acinetobacter baumannii. J Bacteriol 202(2020).

16. Kundra, S., Colomer-Winter, C. \& Lemos, J.A. Survival of the Fittest: The Relationship of (p)ppGpp With Bacterial Virulence. Front Microbiol 11, 601417 (2020).

17. Hobbs, J.K. \& Boraston, A.B. (p)ppGpp and the Stringent Response: An Emerging Threat to Antibiotic Therapy. ACS Infect Dis 5, 1505-1517 (2019).

18. Arenz, S. et al. The stringent factor RelA adopts an open conformation on the ribosome to stimulate ppGpp synthesis. Nucleic Acids Res 44, 6471-81 (2016).

19. Brown, A., Fernandez, I.S., Gordiyenko, Y. \& Ramakrishnan, V. Ribosome-dependent activation of stringent control. Nature 534, 277-80 (2016).

20. Loveland, A.B. et al. Ribosome*RelA structures reveal the mechanism of stringent response activation. Elife 5(2016).

21. Pausch, P. et al. Structural Basis for Regulation of the Opposing (p)ppGpp Synthetase and Hydrolase within the Stringent Response Orchestrator Rel. Cell Rep 32, 108157 (2020).

22. Hogg, T., Mechold, U., Malke, H., Cashel, M. \& Hilgenfeld, R. Conformational antagonism between opposing active sites in a bifunctional RelA/SpoT homolog 
modulates (p)ppGpp metabolism during the stringent response [corrected]. Cell 117, 57-68 (2004).

23. Tamman, H. et al. A nucleotide-switch mechanism mediates opposing catalytic activities of Rel enzymes. Nat Chem Biol 16, 834-840 (2020).

24. Mojr, V. et al. Nonhydrolysable Analogues of (p)ppGpp and (p)ppApp Alarmone Nucleotides as Novel Molecular Tools. ACS Chem Biol (2021).

25. Fitzsimmons, L.F. et al. SpoT Induces Intracellular Salmonella Virulence Programs in the Phagosome. mBio 11(2020).

26. Vogt, S.L. et al. The stringent response is essential for Pseudomonas aeruginosa virulence in the rat lung agar bead and Drosophila melanogaster feeding models of infection. Infect Immun 79, 4094-104 (2011).

27. Mechold, U., Murphy, H., Brown, L. \& Cashel, M. Intramolecular regulation of the opposing (p)ppGpp catalytic activities of Rel(Seq), the Rel/Spo enzyme from Streptococcus equisimilis. J Bacteriol 184, 2878-88 (2002).

28. Takada, H. et al. Ribosome association primes the stringent factor Rel for tRNAdependent locking in the A-site and activation of (p)ppGpp synthesis. Nucleic Acids Res 49, 444-457 (2021).

29. Svitil, A.L., Cashel, M. \& Zyskind, J.W. Guanosine tetraphosphate inhibits protein synthesis in vivo. A possible protective mechanism for starvation stress in Escherichia coli. J Biol Chem 268, 2307-11 (1993).

30. Turnbull, K.J., Dzhygyr, I., Lindemose, S., Hauryliuk, V. \& Roghanian, M. Intramolecular Interactions Dominate the Autoregulation of Escherichia coli Stringent Factor RelA. Front Microbiol 10, 1966 (2019).

31. Winther, K.S., Roghanian, M. \& Gerdes, K. Activation of the Stringent Response by Loading of RelA-tRNA Complexes at the Ribosomal A-Site. Mol Cell 70, 95-105 e4 (2018).

32. Richter, D. Uncharged tRNA inhibits guanosine 3',5'-bis (diphosphate) 3'pyrophosphohydrolase [ppGppase], the spoT gene product, from Escherichia coli. Mol Gen Genet 178, 325-7 (1980).

33. Gropp, M., Strausz, Y., Gross, M. \& Glaser, G. Regulation of Escherichia coli RelA requires oligomerization of the C-terminal domain. J Bacteriol 183, 570-9 (2001).

34. Kaspy, I. \& Glaser, G. Escherichia coli RelA Regulation via Its C-Terminal Domain. Front Microbiol 11, 572419 (2020).

35. Yang, X. \& Ishiguro, E.E. Dimerization of the RelA protein of Escherichia coli. Biochem Cell Biol 79, 729-36 (2001).

36. Roghanian, M. et al. (p)ppGpp controls stringent factors by exploiting antagonistic allosteric coupling between catalytic domains. Mol Cell 81, 3310-3322 e6 (2021).

37. Sinha, A.K. \& Winther, K.S. The RelA hydrolase domain acts as a molecular switch for (p)ppGpp synthesis. Commun Biol 4, 434 (2021).

38. Avarbock, D., Avarbock, A. \& Rubin, H. Differential regulation of opposing RelMtb activities by the aminoacylation state of a tRNA.ribosome.mRNA.RelMtb complex. Biochemistry 39, 11640-8 (2000).

39. Van Nerom, K., Tamman, H., Takada, H., Hauryliuk, V. \& Garcia-Pino, A. The Rel stringent factor from Thermus thermophilus: crystallization and X-ray analysis. Acta Crystallogr F Struct Biol Commun 75, 561-569 (2019).

40. Heinemeyer, E.A., Geis, M. \& Richter, D. Degradation of guanosine 3'-diphosphate 5'diphosphate in vitro by the spoT gene product of Escherichia coli. Eur J Biochem 89, 125-31 (1978).

41. Ronneau, S. et al. Regulation of (p)ppGpp hydrolysis by a conserved archetypal regulatory domain. Nucleic Acids Res 47, 843-854 (2019). 
42. Takada, H. et al. The C-Terminal RRM/ACT Domain Is Crucial for Fine-Tuning the Activation of 'Long' RelA-SpoT Homolog Enzymes by Ribosomal Complexes. Front Microbiol 11, 277 (2020).

43. Kim, K. et al. ppGpp signaling plays a critical role in virulence of Acinetobacter baumannii. Virulence 12, 2122-2132 (2021).

44. Li, J. et al. Genetically tunable frustration controls allostery in an intrinsically disordered transcription factor. Elife 6(2017).

45. Keul, N.D. et al. The entropic force generated by intrinsically disordered segments tunes protein function. Nature 563, 584-588 (2018).

46. Battesti, A. \& Bouveret, E. Acyl carrier protein/SpoT interaction, the switch linking SpoT-dependent stress response to fatty acid metabolism. Mol Microbiol 62, 1048-63 (2006).

47. Lee, J.W., Park, Y.H. \& Seok, Y.J. Rsd balances (p)ppGpp level by stimulating the hydrolase activity of SpoT during carbon source downshift in Escherichia coli. Proc Natl Acad Sci U S A 115, E6845-E6854 (2018).

48. Kruger, L. et al. A meet-up of two second messengers: the c-di-AMP receptor DarB controls (p)ppGpp synthesis in Bacillus subtilis. Nat Commun 12, 1210 (2021). 
648 Fig. 1. A. baumannii SpoT is a monofunctional alarmone hydrolase. (a) Evolution of long 649 RSHs in Proteobacteria. Duplication of the ancestral bifunctional RSH Rel in Beta- and 650 Gammaproteobacterial lineage gave rise to RelA and SpoT, leading to subfunctionalisation of 651 RelA as monofunctional SYNTH-only alarmone synthetase and SpoT as a predominantly-HD 652 RSH. In the Moraxellaceae family of Gammaproteobacteria, SpoT has undergone further 653 subfunctionalisation, evolving into a monofunctional HD-only alarmone hydrolase. (b) 654 Alignment of SYNTH-critical regions in long RSHs highlights the sequence divergence in 655 Moraxellaceae SpoTs. (c) Co-expression of $\mathrm{SpoT}_{A b}$ counteracts the growth defect in ppGpp ${ }^{0}$ 656 ( $\triangle$ relA $\Delta s p o T)$ E. coli caused by RelA expression. This demonstrates that $\mathrm{SpoT}_{A b}$ is HD-active 657 in the E. coli host. (d) While the SYNTH activity of ectopically expressed SpoT Ec $_{\text {is }}$ issential 658 and sufficient for promoting the growth of $\mathrm{ppGpp}^{0}$ E. coli on M9 minimal medium, $\mathrm{SpoT}_{A b}$ 659 fails to promote the growth of $\Delta$ relA $\triangle$ spoT E. coli on M9. This demonstrates that, unlike $660 \mathrm{SpoT}_{E c}$, which is SYNTH-active, $\mathrm{SpoT}_{A b}$ is SYNTH-inactive.

661

\section{2}

Fig. 2. Full-length monomeric $A$. baumannii SpoT adopts a compact "mushroom"-shaped HD-active $\tau$-state. (a) Structure of "mushroom"-shaped SpoT ${ }_{A b}$-ppGpp complex in the $\tau$-state. The domain organization, from $\mathrm{N}$ to $\mathrm{C}$ terminus: NTD domains hydrolase (HD), pseudosynthetase (pseudo-SYNTH) and Core, and CTD domains, TGS, Helical (HEL), Zn-finger (ZFD) and RNA recognition motif (RRM). The ppGpp alarmone is in red. (b) Cartoon representation the $\operatorname{SpoT}_{A b}$. The "stem" of the mushroom is formed by the enzymatic HD domain and the "cap" by the regulatory domains: NTD pseudo-SYNTH domain and the CTD domains. (c) Ribbon representation of the $S \mathrm{ST}_{A b}$-ppGpp complex. The $\alpha 6 / \alpha 7$ motif is held in the hydrolysis-compatible position by the folded Core domain and the TGS $\beta$-hairpin, with the Core domain communicating allosteric signals to HD from the regulatory domains. (d) The HD activity of $\operatorname{SpoT}_{A b}$ is insensitive to the addition of $E$. coli $70 \mathrm{~S}$ ribosomes, and non-specifically weakly inhibited by both aminoacylated and deacyated E. coli tRNA ${ }^{\mathrm{Val}}$. (e) Analytical size exclusion chromatography (SEC) of $\mathrm{SpoT}_{A b}$ supports its monomeric nature in solution. (f) Experimental X-ray scattering (SAXS) analysis of $\mathrm{SpoT}_{A b}$ at $8 \mathrm{mg} / \mathrm{mL}$ further confirms the monomeric nature of $\operatorname{SpoT}_{A b}$. The analysis of the normalised Kratky plot (insert) of the SAXS curve reveals folded globular shape of $\operatorname{SpoT}_{A b}$. (g) Ab initio envelope of $\mathrm{SpoT}_{A b}$ reconstructed from the experimental SAXS data superimposed on the crystal structure. Comparison of both models shows that in solution the enzyme adopts the same conformation as observed in the crystal. 
682 Fig. 3. A. baumannii SpoT is a $\mathrm{Mn}^{2+}$-dependent (p)ppGpp hydrolase. (a) Surface 683 representation of $\operatorname{SpoT}_{A b}$ in the $\tau$-state. The active site cavity in the HD domain is boxed in 684 dashed lines. (b) Zoom into the HD active site of the SpoT $_{A b}$-ppGpp complex. The acidic half 685 of the interface (residues K140, E82, D83, Y51 and R45) and the $\mathrm{Mn}^{2+}$ ion activate the water 686 molecule for nucleophilic attack of the pyrophosphate bond pf ppGpp, while the basic half of 687 the interface (K46, K158 and R161) stabilises the $3^{\prime}$ and 5' phosphates of the alarmone 688 substrate. (c) Ribbon representation of the active site of $\mathrm{SpoT}_{A b}$ revealing the residues involved 689 in coordination of ppGpp. (d) Effects of Ala-substitutions in the ppGpp binding site on the HD 690 activity of $\operatorname{SpoT}_{A b}$. The residues for substitution were selected as per (c). (e) ITC titration of $691 \mathrm{Mn}^{2+}$ into unliganded apo-SpoT $\mathrm{T}_{A b}$. (f) Hydrolase activity of unliganded apo-SpoT $A b$ as a 692 function of increasing concentrations of $\mathrm{Mn}^{2+}$. (g) Structure of the $\mathrm{Mn}^{2+}$-free N-terminal region 693 of $\mathrm{SpoT}_{\mathrm{Ab}}, \mathrm{SpoT}_{A b}{ }^{\mathrm{NTD}}$. The HD domain is in purple and the pseudo-SYNTH is in yellow. The 694 disordered active site is labeled. (h) Superposition of the HD domain of $\operatorname{SpoT}_{A b}$ complexed with $695 \mathrm{ppGpp}$ (in light blue) onto $\mathrm{Mn}^{2+}$-free $\mathrm{SpoT}_{A b}$ (in purple). The key conformation differences in 696 catalytically-crucial active site residues and the structural elements $\alpha 3, \alpha 4$ and $\alpha 8$ are 697 highlighted as dashed arrows and shown in bold, respectively.

699 Fig. 4. The CTD controls the hydrolysis activity of SpoT by controlling the equilibrium 700 between HD-active $\tau$-state and HD-inactive relaxed conformations. (a) The HD 701 functionality test of truncated versions of $\mathrm{SpoT}_{A b}$. $\mathrm{SpoT}_{A b}$ variants were co-expressed expressed 702 with RelA $A b$ in $\Delta r e l A \Delta s p o T$ Ptac::relA A. baumannii (AB5075). The ability of SpoT ${ }_{A b}$ to 703 promote the growth is reflective of its HD competence. (b) Hydrolase activity of SpoT $A b$ and 704 the C-terminally truncated $\operatorname{SpoT}_{A b}$ variants. Turnovers corresponding to each protein variant 705 are coloured as per the domain colour code on (a). (c) Cartoon representation of the allosteric 706 network defined by the Core domain connecting the domains of the enzyme in the $\tau$-state. The 707 key interface residues are shown as sticks and labeled. (d) Hydrolase activity of crucial Core 708 residues involved in interactions with other domains of $\operatorname{SpoT}_{A b}$ (A384R contacting SYNTH, 709 A351K contacting RRM, L356D contacting HD, L373G/D374G contacting ZFD, Y375G 710 contacting the TGS). The TGS:HD interface is also probed with the E379K/W382K point 711 mutant and $\triangle$ TGS-HEL versions. The $\tau$-state stabilising substitutions D374R and 712 I637D/R641D increase the HD activity. (e, f) SAXS curves of L356D in the $\tau$-state (e) or 713 relaxed state (f). (g) Pseudo-atomic model of the relaxed state of $\mathrm{SpoT}_{A b}$ calculated with 714 Dadimodo (Evrard et al., 2011) using the experimental SAXS data from (f). (h) Comparison of 
the experimental SAXS data from the relaxed state of L356D (in grey) with the theoretical scattering curve of the relaxed state (solid line) obtained from the Dadimodo model. (i) SAXS curve of $\operatorname{RelA}_{A b}$ is consistent with the dimensions of the relaxed state. (j, k) SAXS curves of $\operatorname{Rel}_{B s}$ in the $\tau$-state (j) or relaxed state (k). (l) Cartoon representation of experimentally observed conformational states as well as particle dimensions of long RSH enzymes.

Fig. 5. The Core domain of SpoT transduces the allosteric signal from the regulatory CTD and pseudo-SNTH to the enzymatic HD domain. (a, b) Cartoon representation of the interactions stabilising the $\alpha 6-\alpha 7$ motif of the HD active site (A). While the Core wraps around $\alpha 7$, the TGS $\beta$-hairpin forms a small hydrophobic patch that stabilises $\alpha 6$. These interactions preclude the movement of $\alpha 6-\alpha 7$ and maintain $\operatorname{SpoT}_{A b}$ in a constitutive hydrolase-primed state. Key interface residues are shown as sticks and labelled. (b) The experimental SAXS curve of $\mathrm{SpoT}_{A b}{ }^{\mathrm{E} 379 \mathrm{~K} / \mathrm{W} 382 \mathrm{~K}}$ is consistent with the dimensions of the $\tau$-state. Cartoon representation of the HD:Core:RRM signal transduction axis. (c) The architecture of the $\tau$-state suggests that the RRM is locked in place via the Core and supporting interaction provided by pseudo-SYNTH, suggesting that additional tethering of RRM to pseudo-SYNTH could further stabilise the $\tau$ conformation. (d) SAXS curve of the $\operatorname{SpoT}_{A b}{ }^{1637 \mathrm{D} / \mathrm{R} 641 \mathrm{D}}$ variant in which substitutions I637D/R641D and I637A/R641A promote H-bonding and stabilise the $\alpha$-helical structure, respectively, is consistent with the dimensions of the $\tau$-state. (e) In vivo HD functionality tests of $\mathrm{SpoT}_{A b}$ variants D374G and I637D/R641D expressed from the inducible Ptac promoter in a $\Delta$ relA $\Delta$ spoT background of $A$. baumannii (AB5075) expressing relA from a replicative plasmid (pPrelA::relA). The stabilising substitutions D374R and I637D/R641D phenocopy the WT. (f) Virulence assays in the G. mellonella infection model demonstrate the essentiality of intact allosteric regulation of $\mathrm{SpoT}_{A b}$ for virulence. G. mellonella larvae were injected with $\approx 2 \times 10$ CFU of A. baumannii (AB5075) strains $\left(10 \mu \mathrm{l}\right.$ at $\left.\approx 2 \times 10^{7} \mathrm{CFU} / \mathrm{mL}\right)$, eight larvae were inoculated per strain, and incubated at $37^{\circ} \mathrm{C}$ in the dark. The viability of the larvae was scored every $24 \mathrm{~h}$.

Fig. 6. The enzymatic output of subfunctionalised RelA and SpoT RSH enzymes is evolutionarily tuned through constrains of the conformational landscape. (a) Control of the enzymatic output of the ancestral bifunctional Rel[HS]. Upon amino acid starvation Rel is recruited to starved ribosomal complexes. The ribosome-bound Rel assumes an extended conformation in which the auto-inhibitory effect of the CTD region on the SYNTH activity is released. The full activation of SYNTH activity is achieved upon binding of (p)ppGpp to an 
749 allosteric site within the NTD and release of the SYNTH inhibition by the HD domain. 750 Conversely, off the ribosome the enzyme assumes the $\tau$-state. In this conformation locking of 751 the $\alpha 6-\alpha 7$ motif by the CTD organises the HD active site residues to promote the HD activity. 752 This, in turn, strongly inhibits the SYNTH activity via inter-NTD regulation. The full activation 753 of either SYNTH or HD requires allosteric signalling from CTD to NTD enzymatic domains. 754 (b, c) Evolution of SpoT as a predominantly dedicated hydrolase involved the loss of the 755 allosteric control of the NTD by (p)ppGpp as well as by the ribosome. In bifunctional SpoT[HS] 756 present in the majority of Gamma- and Betaproteobacteria, while the equilibrium is strongly 757 shifted towards the HD-active $\tau$-state, the enzyme is capable of inefficient (p)ppGpp synthesis 758 in the relaxed state (B). Subfunctionalisation of SpoT in Moraxellaceae has resulted in the 759 monofunctional hydrolase SpoT[Hs], which naturally populates only the compact $\tau$-state and is 760 SYNTH-inactive. (d) Subfunctionalisation of Gamma- and Betaproteobacterial RelA[hS] 761 constitutes the other extreme case of evolutionary restriction of the conformational dynamics 762 of the ancestral Rel[HS]. While losing its HD activity, RelA retains all the allosteric regulatory 763 elements of Rel. Being a dedicated (p)ppGpp synthetase enzyme, off the ribosome RelA does 764 not assume the $\tau$-state. Instead, it predominantly populates the functionally frustrated resting 765 state equivalent to the relaxed state of Rel, primed to assume the elongated ribosome-associated 766 state triggered by the 70S ribosome, uncharged tRNA and alarmones during stringency. Red 767 circles represent inhibited catalytic centres, green circles represent fully activated catalytic 768 centres, and dashed green circles represent idling catalytic centres. 


\section{Figures}

\section{Figure 1}

A. baumannii SpoT is a monofunctional alarmone hydrolase. (a) Evolution of long RSHs in Proteobacteria. Duplication of the ancestral bifunctional RSH Rel in Beta- and Gammaproteobacterial lineage gave rise to RelA and SpoT, leading to subfunctionalisation of RelA as monofunctional SYNTHonly alarmone synthetase and SpoT as a predominantly-HD RSH. In the Moraxellaceae family of Gammaproteobacteria, SpoT has undergone further subfunctionalisation, evolving into a monofunctional HD-only alarmone hydrolase. (b) Alignment of SYNTH-critical regions in long RSHs highlights the sequence divergence in Moraxellaceae SpoTs. (c) Co-expression of SpoTAb counteracts the growth defect in ppGpp0 (DrelA DspoT) E. coli caused by RelA expression. This demonstrates that SpoTAb is HDactive in the E. coli host. (d) While the SYNTH activity of ectopically expressed SpoTEc is essential and sufficient for promoting the growth of ppGpp0 E. coli on M9 minimal medium, SpoTAb fails to promote the growth of DrelA DspoT E. coli on M9. This demonstrates that, unlike SpoTEc, which is SYNTH-active, SpoTAb is SYNTH-inactive.

\section{Figure 2}

Full-length monomeric A. baumannii SpoT adopts a compact "mushroom"-shaped HD-active T-state. (a) Structure of "mushroom"-shaped SpoTAb-ppGpp complex in the t-state. The domain organization, from N to $C$ terminus: NTD domains hydrolase (HD), pseudo synthetase (pseudo-SYNTH) and Core, and CTD domains, TGS, Helical (HEL), Zn-finger (ZFD) and RNA recognition motif (RRM). The ppGpp alarmone is in red. (b) Cartoon representation the SpoTAb. The "stem" of the mushroom isformed by the enzymatic HD domain and the "cap" by the regulatory domains: NTD pseudo-SYNTH domain and the CTD domains. (c) Ribbon representation of the SpoTAb-ppGpp complex. The a6/a7 motif is held in the hydrolysiscompatible position by the folded Core domain and the TGS $\beta$-hairpin, with the Core domain communicating allosteric signals to HD from the regulatory domains. (d) The HD activity of SpoTAb is insensitive to the addition of E. coli 70S ribosomes, and non-specifically weakly inhibited by both aminoacylated and deacyated E. coli tRNAVal. (e) Analytical size exclusion chromatography (SEC) of SpoTAb supports its monomeric nature in solution. (f) Experimental X-ray scattering (SAXS) analysis of SpoTAb at $8 \mathrm{mg} / \mathrm{mL}$ further confirms the monomeric nature of SpoTAb. The analysis of the normalised Kratky plot (insert) of the SAXS curve reveals folded globular shape of SpoTAb. (g) Ab initio envelope of SpoTAb reconstructed from the experimental SAXS data superimposed on the crystal structure. Comparison of both models shows that in solution the enzyme adopts the same conformation as observed in the crystal. 


\section{Figure 3}

A. baumannii SpoT is a Mn2+-dependent (p)ppGpp hydrolase. (a) Surface representation of SpoTAb in the $\tau$-state. The active site cavity in the HD domain is boxed in dashed lines. (b) Zoom into the HD active site of the SpoTAb-ppGpp complex. The acidic half of the interface (residues K140, E82, D83, Y51 and R45) and the Mn2+ ion activate the water molecule for nucleophilic attack of the pyrophosphate bond pf ppGpp, while the basic half of the interface (K46, K158 and R161) stabilises the 3 ' and 5 ' phosphates of the alarmone substrate. (c) Ribbon representation of the active site of SpoTAb revealing the residues involved in coordination of ppGpp. (d) Effects of Ala-substitutions in the ppGpp binding site on the HD activity of SpoTAb. The residues for substitution were selected as per (c). (e) ITC titration of Mn2+ into unliganded apo-SpoTAb. (f) Hydrolase activity of unliganded apo-SpoTAb as a function of increasing concentrations of Mn2+. (g) Structure of the Mn2+-free N-terminal region of SpoTAb, SpoTAbNTD. The HD domain is in purple and the pseudo-SYNTH is in yellow. The disordered active site islabeled. (h) Superposition of the HD domain of SpoTAb complexed with ppGpp (in light blue) onto Mn2+-free SpoTAb (in purple). The key conformation differences in catalytically-crucial active site residues and the structural elements a3, a4 and a8 are highlighted as dashed arrows and shown in bold, respectively.

\section{Figure 4}

The CTD controls the hydrolysis activity of SpoT by controlling the equilibrium between HD-active T-state and HD-inactive relaxed conformations. (a) The HD functionality test of truncated versions of SpoTAb. SpoTAb variants were co-expressed expressed with RelAAb in $\triangle$ relA $\triangle$ spoT Ptac::relA A. baumannii (AB5075). The ability of SpoTAb to promote the growth is reflective of its HD competence. (b) Hydrolase activity of SpoTAb and the C-terminally truncated SpoTAb variants. Turnovers corresponding to each protein variant are coloured as per the domain colour code on (a). (c) Cartoon representation of the allosteric network defined by the Core domain connecting the domains of the enzyme in the $\tau$-state. The key interface residues are shown as sticks and labeled. (d) Hydrolase activity of crucial Core residues involved in interactions with other domains of SpoTAb (A384R contacting SYNTH, A351K contacting RRM, L356D contacting HD, L373G/D374G contacting ZFD, Y375G contacting the TGS). The TGS:HD interface is also probed with the $\mathrm{E} 379 \mathrm{~K} / \mathrm{W} 382 \mathrm{~K}$ point mutant and $\triangle \mathrm{TGS}-\mathrm{HEL}$ versions. The $\mathrm{t}$-state stabilising substitutions D374R and 1637D/R641D increase the HD activity. (e, f) SAXS curves of L356D in the $\mathrm{t}$-state (e) or relaxed state (f). (g) Pseudo-atomic model of the relaxed state of SpoTAb calculated with Dadimodo (Evrard et al., 2011) using the experimental SAXS data from (f). (h) Comparison of 22 the experimental SAXS data from the relaxed state of L356D (in grey) with the theoretical scattering curve of the relaxed state (solid line) obtained from the Dadimodo model. (i) SAXS curve of RelAAb is consistent with the dimensions of the relaxed state. $(j, k)$ SAXS curves of RelBs in the $t$-state $(j)$ or relaxed state $(k)$. 
(I) Cartoon representation of experimentally observed conformational states as well as particle dimensions of long RSH enzymes.

\section{Figure 5}

The Core domain of SpoT transduces the allosteric signal from the regulatory CTD and pseudo-SNTH to the enzymatic HD domain. $(a, b)$ Cartoon representation of the interactions stabilising the a6-a7 motif of the HD active site (A). While the Core wraps around $\mathrm{a} 7$, the TGS $\beta$-hairpin forms a small hydrophobic patch that stabilises a6. These interactions preclude the movement of a6-a7 and maintain SpoTAb in a constitutive hydrolase-primed state. Key interface residues are shown as sticks and labelled. (b) The experimental SAXS curve of SpoTAbE379K/W382K is consistent with the dimensions of the $\mathrm{t}$-state. Cartoon representation of the HD:Core:RRM signal transduction axis. (c) The architecture of the $\mathrm{t}$-state suggests that the RRM is locked in place via the Core and supporting interaction provided by pseudoSYNTH, suggesting that additional tethering of RRM to pseudo-SYNTH could further stabilise the tconformation. (d) SAXS curve of the SpoTAbI637D/R641D variant in which substitutions 1637D/R641D and I637A/R641A promote $\mathrm{H}$-bonding and stabilise the a-helical structure, respectively, is consistent with the dimensions of the $T$-state. (e) In vivo HD functionality tests of SpoTAb variants D374G and 1637D/R641D expressed from the inducible Ptac promoter in a $\Delta$ relA $\Delta$ spoT background of $A$. baumannii (AB5075) expressing relA from a replicative plasmid (pPrelA::relA). The stabilising substitutions D374R and 1637D/R641D phenocopy the WT. (f) Virulence assays in the G. mellonella infection model demonstrate the essentiality of intact allosteric regulation of SpoTAb for virulence. G. mellonella larvae were injected with $\approx 2 \times 10 \mathrm{CFU}$ of $A$. baumannii (AB5075) strains $(10 \mu \mathrm{l}$ at $\approx 2 \times 107 \mathrm{CFU} / \mathrm{mL}$ ), eight larvae were inoculated per strain, and incubated at $37^{\circ} \mathrm{C}$ in the dark. The viability of the larvae was scored every $24 \mathrm{~h}$.

\section{Figure 6}

The enzymatic output of subfunctionalised RelA and SpoT RSH enzymes is evolutionarily tuned through constrains of the conformational landscape. (a) Control of the enzymatic output of the ancestral bifunctional Rel[HS]. Upon amino acid starvation Rel is recruited to starved ribosomal complexes. The ribosome-bound Rel assumes an extended conformation in which the auto-inhibitory effect of the CTD region on the SYNTH activity is released. The full activation of SYNTH activity is achieved upon binding of (p)ppGpp to an 23 allosteric site within the NTD and release of the SYNTH inhibition by the HD domain. Conversely, off the ribosome the enzyme assumes the t-state. In this conformation locking of the a6- $a 7$ motif by the CTD organises the HD active site residues to promote the HD activity. This, in turn, strongly inhibits the SYNTH activity via inter-NTD regulation. The full activation of either SYNTH or HD requires allosteric signalling from CTD to NTD enzymatic domains. (b, c) Evolution of SpoT as a predominantly dedicated hydrolase involved the loss of the allosteric control of the NTD by (p)ppGpp as 
well as by the ribosome. In bifunctional SpoT[HS] present in the majority of Gamma- and Betaproteobacteria, while the equilibrium is strongly shifted towards the HD-active $\tau$-state, the enzyme is capable of inefficient (p)ppGpp synthesis in the relaxed state (B). Subfunctionalisation of SpoT in Moraxellaceae has resulted in the monofunctional hydrolase SpoT[Hs], which naturally populates only the compact t-state and is SYNTH-inactive. (d) Subfunctionalisation of Gamma- and Betaproteobacterial RelA[hS] constitutes the other extreme case of evolutionary restriction of the conformational dynamics of the ancestral Rel[HS]. While losing its HD activity, RelA retains all the allosteric regulatory elements of Rel. Being a dedicated (p)ppGpp synthetase enzyme, off the ribosome RelA does not assume the $T$-state. Instead, it predominantly populates the functionally frustrated resting state equivalent to the relaxed state of Rel, primed to assume the elongated ribosome-associated state triggered by the 70S ribosome, uncharged tRNA and alarmones during stringency. Red circles represent inhibited catalytic centres, green circles represent fully activated catalytic centres, and dashed green circles represent idling catalytic centres.

\section{Supplementary Files}

This is a list of supplementary files associated with this preprint. Click to download.

- SupplementalDataDec.xlsx

- D1292120009valreportfullP1.pdf

- Tammanv260nlinemethods.pdf

- Tammanv26SupplementalFigures.pdf

- Tammanv26SupplementalTables.pdf

- abuSpoTppGppfinalaltgood.pdf

- nreditorialpolicychecklistNCHEMBA220114337.pdf 